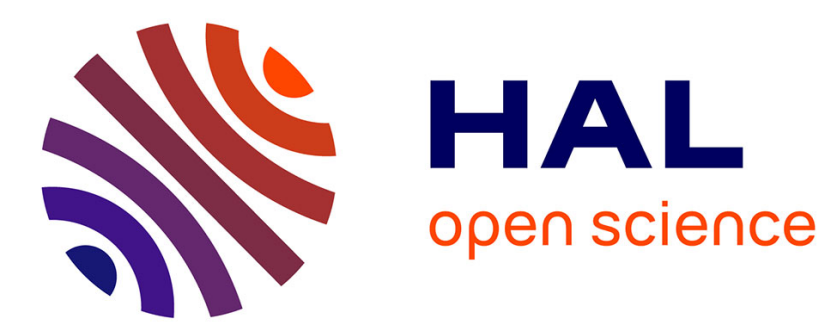

\title{
Compaction of elastic granular materials: inter-particles friction effects and plastic events
}

\author{
Thi-Lo Vu, Saeid Nezamabadi, Serge Mora
}

\section{To cite this version:}

Thi-Lo Vu, Saeid Nezamabadi, Serge Mora. Compaction of elastic granular materials: inter-particles friction effects and plastic events. Soft Matter, 2020, 16 (3), pp.679-687. 10.1039/C9SM01947B . hal-02404009

\section{HAL Id: hal-02404009 \\ https://hal.science/hal-02404009}

Submitted on 11 Dec 2019

HAL is a multi-disciplinary open access archive for the deposit and dissemination of scientific research documents, whether they are published or not. The documents may come from teaching and research institutions in France or abroad, or from public or private research centers.
L'archive ouverte pluridisciplinaire HAL, est destinée au dépôt et à la diffusion de documents scientifiques de niveau recherche, publiés ou non, émanant des établissements d'enseignement et de recherche français ou étrangers, des laboratoires publics ou privés. 


\title{
Compaction of elastic granular materials: inter-particles friction effects and plastic events $\dagger$
}

\author{
Thi-Lo Vu, ${ }^{\text {abc }}$ Saeid Nezamabadi ${ }^{c d}$ and Serge Mora $\quad * c$
}

The uni-axial compaction of granular materials made of elastic neo-Hookean particles is investigated in the quasi-static regime. Two-dimensional disk assemblies are simulated using the Finite Element model coupled with Contact Dynamics method for dealing both with finite deformations of the particles and contact interactions. Due to large deformations of the particles, the packing fraction of the system increases continuously during the compaction process, reaching values close to 1 . The influence of the coefficient of friction between the particles on the macroscopic and micro-structural behaviors of the system is thoroughly discussed.

\section{Introduction}

Granular materials made of rigid or semi-rigid particles have been widely studied experimentally and numerically in the past, leading a comprehensive understanding of their mechanical properties. The compaction of solid particle assemblies is an important industrial problem since it is involved in a wide range of processes such as pharmaceutical tableting, ${ }^{1-3}$ powder metallurgy $^{4-6}$ and ceramics. ${ }^{7-10}$ Since high pressures are often involved during compaction, the confining stresses can be on the order of the elastic modulus of the particles or of their yield stress. In this case, the solid particles cannot be anymore considered as rigid or semi-rigid: even if the initial shapes of the particles are spherical, these shapes continuously evolve during the compression and become non-spherical.

In order to model soft granular systems, a simple approach is to use the Discrete Element Method (DEM) by considering the deformable contacts between particles for which the contact force and deformation are deduced from contact laws depending on the interpenetration of the particles (e.g. Hertzian contact law). ${ }^{11-14}$ This approach is hence based on small deflections of the particle surface. However, accounting the large deformations of soft particles requires to introduce the internal degrees of freedom with an appropriate contact treatment. In this context, the first simple strategy is the Bonded Particle Model (BPM),

\footnotetext{
${ }^{a}$ Division of Computational Mathematics and Engineering, Institute for Computational Science, Ton Duc Thang University, Ho Chi Minh City, Vietnam

${ }^{b}$ Faculty of Civil Engineering, Ton Duc Thang University, Ho Chi Minh City, Vietnam. E-mail: vuthilo@tdtu.edu.vn

${ }^{c}$ LMGC, Université de Montpellier, CNRS, Montpellier, France. E-mail: saied.nezamabadi@umontpellier.fr, serge.mora@umontpellier.fr

${ }^{d}$ IATE, CIRAD, INRA, Montpellier SupAgro, Université de Montpellier, F-34060, Montpellier, France

$\dagger$ PACS number: 83.80.Fg,46.25.-y,87.10.K
}

which consists in discretizing each particle as an aggregate of rigid primary particles with cohesive interactions. A soft particle can hence deform as a result of the relative motions of its primary particles while staying together as a solid particle. ${ }^{15}$ Another approach allowing taking into account the realistic behavior of soft particles, is based on an implicit Material Point Method (MPM) coupled with the Contact Dynamics (CD) method. ${ }^{15-17}$ In the MPM, each particle is discretized by a set of material points and the implicit MPM formulation permits to couple efficiently with implicit modeling of unilateral contacts and friction between particles as in the CD method. ${ }^{18,19}$ An alternative technique consists in combining the Finite Element Method (FEM) with DEM. ${ }^{20,21}$ In this case, each particle is discretized by the FEM and interactions between particles are modeled by DEM. As an application example, Gethin et al. (2001) ${ }^{22}$ applied this technique to the compaction of metal powders.

In this paper, our numerical approach is based on the FEM-CD coupling. Since here, we consider neo-Hookean rubber-like particles undergoing finite elastic (reversible) strains, the FEM formulation is based on the finite strain theory. ${ }^{23}$ In the previous works, ${ }^{24,25}$ by using this approach, numerical simulations of the uni-axial compression of a system of 100 rubber-like cylindrical particles have been presented. The numerical results have been successfully compared with experiments. In these simulations, the relatively low number of particles and the coefficient of friction were imposed by the experiments, as well as the range of the explored packing fractions (smaller than $88 \%$ ). Here, we go further by carrying out a parametric numerical study of the inter-particles friction effect during an uni-axial compaction of a similar system (made of non-Brownian particles that can undergo large elastic deformation), but containing a larger amount of particles (400) in order to minimize finite size effects. ${ }^{26}$

The paper is organized as follows. Details about the granular system and the numerical method are given in Section II. 
Section III concerns the analysis of the stress applied to the system boundary during the compaction in a wide range of packing fractions, from the rigidity transition to packing fractions close to 1 . The stress distribution inside the system is investigated in Section IV. Finally, the role of the inter-particles coefficient of friction on plastic events occurring in the system during the compaction is highlighted and discussed in Section V. Section VI provides a short summary and discussion.

\section{Methods}

\section{A. Description of the system}

The systems investigated in this paper consist of sets of 400 cylindrical elastic particles with parallel axis (aligned along axis $Z$ ) placed in a box (width $W=0.42 \mathrm{~m}$ along axis $X$ and initial length $L=0.51 \mathrm{~m}$ along axis $Y$, and height $H$ along axis $Z$ ) with rigid walls; see Fig. 1 . One of the two walls parallel to the $X Z$ plane, denoted later as the top wall, is mobile: it moves inward the box with an imposed constant velocity $v$. Since the other walls are fixed, the granular system undergoes a uni-axial compression. The wall opposite to the mobile one, located at $Y=0$, is denoted as the bottom wall. The particle diameters are set in the range $\left[D_{\min }: D_{\max }\right]$ with $D_{\min }=0.01 \mathrm{~m}$ and $D_{\max }=$ $0.015 \mathrm{~m}$ and their height is $H$. The particle size distribution is uniform by particle volume fractions; i.e. all size classes have the same volume of particles so that crystallization is inhibited. The initial configurations are defined by randomly distributing the particle positions in the $X Y$ plane of the box with the initial size of $L \times W$.

In the following, the plane-strain conditions in the $X Y$ plane are considered, assuming the system is invariant along $Z$.

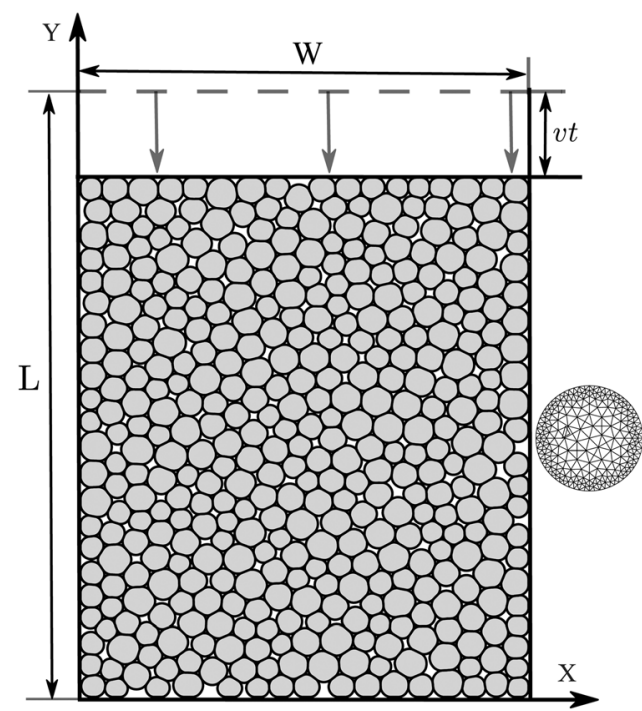

Fig. 1 A 2D assembly of 400 cylindrical particles subjected to uniaxial compression. The granular material lays in a rectangular box with the width $W=0.42 \mathrm{~m}$ and the initial length $L=0.51 \mathrm{~m}$. The top wall moves down with a constant velocity of $v$ during time $t$. Axis $X$ is set parallel to the mobile wall and axis $Y$ is in the direction of the velocity $v$. A typical finite element mesh of a particle is shown on the right.
The packing fraction, defined as the ratio of the volume of particles to the actual system volume, is then determined by the ratio between the cumulative surface of the particle cross sections and the actual box area in the $X Y$ plane. Here, the packing fraction of the initial configuration is chosen to be equal to $\Phi=0.80$. Note that the effects of gravity, surface tension $^{27,28}$ and adhesion ${ }^{29}$ are neglected.

\section{B. Numerical model}

1. General approach. The numerical technique used in this work, which couples the FEM and CD methods and was implemented in the LMGC90 code, ${ }^{30}$ has been described and validated in a previous paper. ${ }^{25}$ To model the system presented above, two-dimensional simulations are performed by considering an equivalent system defined by reducing the cylindrical particles to circular disks and the box to a rectangular area with 3 rigid fixed lines and a rigid mobile line as walls. In the simulations, these walls are modeled as rigid bodies. Moreover, in order to compute more precisely the contact between particles, and between walls and particles as well as to optimize the computational cost, each particle is discretized using a mesh which is denser at the periphery than in its central region; see Fig. 1. It hence reduces the number of degrees of freedom of the system. In this manner, each particle is meshed with about 400, 3-nodes triangular elements.

In the simulations, the contact is considered to be frictional between particles and frictionless between walls and particles. The later reduces the occurrence of arches between the lateral walls $^{31}$ and of stress gradients, ${ }^{32}$ minimizing wall effects. The inter-particles friction is assumed to follow Coulomb friction law. For each simulation, the same coefficient of friction $\mu_{\mathrm{f}}$ between all particles is considered. Moreover, to perform a statistical analysis, each simulation with the same physical parameters was performed using three equivalent different initial configurations.

2. Material behavior of the particles. All the particles are assumed to have the same material behavior and to follow the isotropic neo-Hookean constitutive law. ${ }^{23}$ The bulk mechanical behavior of an elastic body can be described by a strain energy density function. For a neo-Hookean material, the strain energy density function is given by: ${ }^{33,34}$

$$
\Psi=\frac{\mu}{2}\left(I_{1}-3\right)-\mu \ln J+\frac{\lambda}{2}(\ln J)^{2},
$$

with $I_{1}=\operatorname{Tr}\left(\mathbf{F}^{T} \mathbf{F}\right)$ and $J=\operatorname{det}(\mathbf{F})$. F denotes the deformation gradient tensor defined as $\mathbf{F}=\mathbf{I}+\nabla \mathbf{u}$ (I being the second-order identity tensor and $\mathbf{u}$ the displacement field). $\lambda$ and $\mu$ are the Lamé's parameters and $\mu$ denotes also the shear modulus. The Poisson's ratio $\nu$ is related to $\lambda$ and $\mu$ via:

$$
\nu=\lambda / 2(\lambda+\mu) .
$$

Here, we wish to consider incompressible particles $(\nu=0.5)$. For simplifying the numerical procedure, the Poisson's ratio is fixed to $\nu=0.495$. Taking a Poisson's ratio slightly lower than 0.5 makes the particle almost incompressible without significant changes compared to the case of incompressible particles. Otherwise, with $\nu=0.5$, the Lamé's first parameter $\lambda$ would be infinity 
(see eqn (2)) and a Lagrange multiplier algorithm can be used for the simulation.

3. Quasi-static condition. The shear wave velocity, $c=$ $\sqrt{\mu / \rho}$ with $\rho$ the mass density of the particles, is a characteristic velocity of the (quasi-)incompressible elastic material. The time needed for the stress to propagate from one side to an other side of the system scales as $L / c$ with $L$ the length of the system. On the other hand, the characteristic timescale for the load in the system is $L / v$. Hence, the propagation time is negligible compared to the loading characteristic time if $v \ll c$.

In the simulations, we take for the shear modulus a constant value consistent with rubber-like elastomer, fixed to $\mu=0.15$ $\mathrm{MPa}$. The corresponding mass density for such material is $\rho=1180 \mathrm{~kg} \mathrm{~m}^{-3,24,34}$ hence, $c=\sqrt{\mu / \rho} \sim 10 \mathrm{~m} \mathrm{~s}^{-1}$. In the following, the simulations with applied velocities of $0.005 \mathrm{~m} \mathrm{~s}^{-1}, 0.01 \mathrm{~m} \mathrm{~s}^{-1}$ and $0.035 \mathrm{~m} \mathrm{~s}^{-1}$ are carried out, accordingly to the condition established above.

Moreover, to reinforce the quasi-static condition of the simulations, a Rayleigh damper model is considered with the relative damping Rayleigh coefficients of $\alpha=35 \mathrm{~s}^{-1}$ and $\beta=1 \times$ $10^{-4} \mathrm{~s}$. The time step in the simulations is $\delta t=9 \mu \mathrm{s}$. For more details about the choice of these parameters, see a previous work. ${ }^{25}$

\section{Stress transmission}

We consider uni-axial and quasi-static compressions of the granular system, as described above. The mean value of the $y y$ component of the Cauchy stress tensor at the bottom of the box, $\left\langle\sigma_{y y}\right\rangle_{Y=0}$, is shown as a function of the packing fraction $\Phi$ for four coefficients of friction $\mu_{\mathrm{f}}$, in Fig. 2. $\left\langle\sigma_{y y}\right\rangle_{Y=0}$ is computed by dividing the contact forces acting on the bottom wall by the length of this wall. The error bars in Fig. 2 represent the statistical variability of $\left\langle\sigma_{y y}\right\rangle_{Y=0}$ obtained from the three initial equivalent configurations. The small error bar amplitudes show that, although the number of particles (400) is limited, the simulations are able to produce significant and

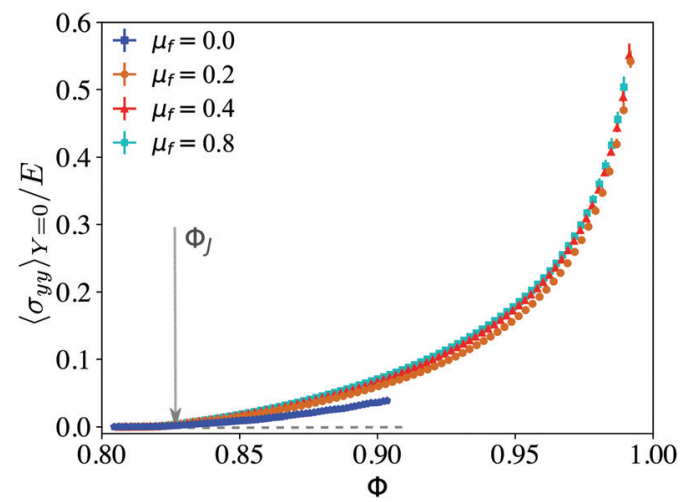

Fig. 2 Mean value of the normal stress applied by the granular system to the bottom wall, $\left\langle\sigma_{y y}\right\rangle_{Y=0}$, normalized by the Young's modulus $E$ of the particles, as a function of the packing fraction $\Phi$ for four coefficients of friction $\mu_{\mathrm{f}}$. The arrow indicates the value $\Phi_{J}\left(\mu_{\mathrm{f}}=0\right)$ of the packing fraction at the rigidity transition for $\mu_{f}=0$. The error bars for $\left\langle\sigma_{y y}\right\rangle_{Y=0}$ are determined by the statistical error of the three initial equivalent configurations. representative values of the transmitted stress, independent of the initial configuration.

For each value of the coefficient of friction $\mu_{\mathrm{f}},\left\langle\sigma_{y y}\right\rangle_{Y=0}$ first fluctuates close to zero below a critical value $\Phi_{J}$ of the packing fraction, that depends on the coefficient of friction $\mu_{\mathrm{f}}$. These fluctuations can be explained by the inertial particle movements: at low packing fractions, the particles are free to move before being totally damped by the Rayleigh damper and wallparticle collisions may occur, inducing sometimes a non zero force at the bottom wall. For $\Phi>\Phi_{J}$ the values of $\left\langle\sigma_{y y}\right\rangle_{Y=0}$ obtained for $v=0.01 \mathrm{~m} \mathrm{~s}^{-1}$ and $v=0.005 \mathrm{~m} \mathrm{~s}^{-1}$ are indistinguishable whereas the ones for $v=0.035 \mathrm{~m} \mathrm{~s}^{-1}$ slightly differ for $\Phi$ close to $\Phi_{J}$. This is again due to few free particles having non zero velocity and striking the bottom wall. Reducing the top wall velocity minimizes these strikes and hence, the forces involved in these rebounds at the bottom wall. In this case, the stress due to impacts is negligible compared to the stress due to the static contact forces applied by the other particles.

Beyond $\Phi_{J},\left\langle\sigma_{y y}\right\rangle_{Y=0}$ increases with $\Phi$ : the stress applied to the top wall is transmitted by the particles to the bottom wall. $\Phi_{J}$ is hence the packing fraction at the rigidity transition. ${ }^{35-38}$ As already observed in ref. 39-42, the packing fraction at the rigidity transition decreases as $\mu_{\mathrm{f}}$ increases: the relative displacement of the particles is reduced by the friction, and the distribution of the particles over the new available area is more easily blocked. For the high values of $\Phi$, the increase rate of $\left\langle\sigma_{y y}\right\rangle_{Y=0}$ with respect to the packing fraction $\Phi$ also increases, and ultimately diverges due to the (quasi-)incompressibility of the particles. Indeed, once all the space of the rigid box is filled by the deformed particles, no compaction is possible anymore. Moreover, for any value of $\Phi,\left\langle\sigma_{y y}\right\rangle_{Y=0}$ is larger as the coefficient of friction $\mu_{\mathrm{f}}$ is larger. It can be again explained by reducing the particle rearrangements due to the friction.

Fig. 3 shows $\left\langle\sigma_{y y}\right\rangle_{Y=0}$ as a function of $\Phi-\Phi_{J}$ in log-log-scales. We observe that $\left\langle\sigma_{y y}\right\rangle_{Y=0}$ well follows a power law $\left\langle\sigma_{y y}\right\rangle_{Y=0} \sim\left(\Phi-\Phi_{J}\right)^{\alpha}$ over a wide range of packing fractions. Such a power law has already

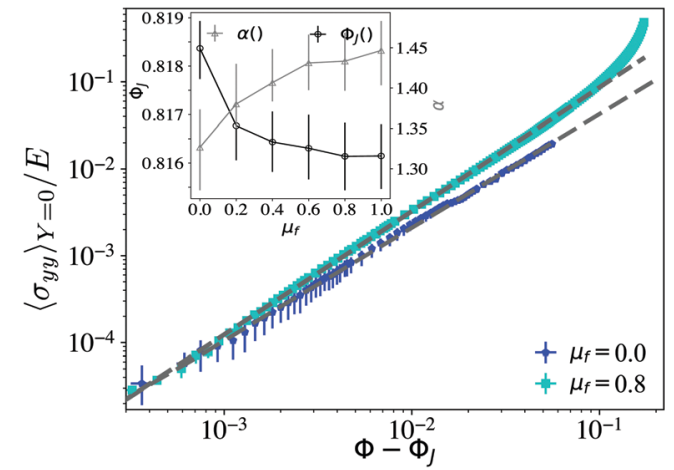

Fig. 3 Mean value of the normal stress applied by the granular system to the bottom wall, $\left\langle\sigma_{y y}\right\rangle_{Y=0}$, normalized by the Young's modulus $E$ of the particles, as a function of the excess packing fraction $\Phi-\Phi_{J}$, in log-log scales, for two values of the coefficient of friction ( $\mu_{\mathrm{f}}=0$ and $\mu_{\mathrm{f}}=0.8$ ). The dashed lines are the power-law fits of $K\left(\Phi-\Phi_{J}\right)^{\alpha}$ with $K$ and $\alpha$ as the fitting parameters. Inset: Values of $\Phi_{J}$ (left axis) and $\alpha$ (right axis) as a function of the coefficient of friction $\mu_{\mathrm{f}}$. The error bars are determined by the statistical error of the three initial configurations. 
been demonstrated in experiments ${ }^{39,43}$ and numerical simulations in bi-axial compaction, but in a far smaller range of packing fractions. ${ }^{11,39}$ In our simulations, the exponent $\alpha$ is found to decrease with the coefficient of friction $\mu_{\mathrm{f}}$. It varies from $\alpha=1.32 \pm 0.4$ for $\mu_{\mathrm{f}}=0$ to the asymptotic value of $\alpha=1.45 \pm 0.4$ for $\mu_{\mathrm{f}}>0.6$. These values of $\alpha$ are different from those obtained by other authors in a bi-axial experiment with a limited resolution of the force measurement, ${ }^{39,43}$ and are also different from simulations done by assuming first order infinitesimal deformations. ${ }^{11,44}$ These discrepancies may arise from the differences in the loading geometry, the system size, or the size polydispersity. In addition, the validity of the approximation of infinitesimal (Hookean) elasticity made in the previous studies is doubtful. Even close to the rigidity transition, since the stress is generated by a small amount of particles within the principal chain forces, ${ }^{45}$ the deformation of these particles are finite. Hence, simulations taking properly into account the finite deformations of the particles are required even for the determination of a scaling law close to the rigidity transition. The exponents found in the literature are close to 1 , which is the well-known value predicted for a Hertzian contact between two particles, i.e. for small deformations. The rigorous treatment of the large deformations leads here to significantly higher exponents, at least for neoHookean particles. Strikingly, the power laws found here are good up to $93 \%$ packing fractions (Fig. 3). The divergence in $\sigma_{y y}$ for $\Phi \simeq 1$ mentioned above naturally implies that the scaling laws are no longer applicable near the maximum packing fraction. For the packing fractions larger than 0.93, one leaves the domain in which $\left\langle\sigma_{y y}\right\rangle_{Y=0}$ follows the power law to enter a new domain with a stiffer increase of $\left\langle\sigma_{y y}\right\rangle_{Y=0}$ as the packing fraction increases.

\section{Local stress}

\section{A. Mean values of the Cauchy stress}

In the simulations, the Cauchy stress tensor $\sigma$ can be computed at any point inside the particles, allowing obtaining its mean value in the whole system. Having the frictionless contact at the walls, the values of $\left\langle\sigma_{y y}\right\rangle_{Y=0}$ and $\left\langle\sigma_{y y}\right\rangle$ are equal except close to the rigidity transition point because of the dynamics effects discussed in Section III. In the following, we do not focus anymore on the rigidity transition, but on high packing fractions for which no difference between the two stresses is relevant.

Fig. 4 displays $\left\langle\sigma_{y y}\right\rangle$ as a function of the mean value of the $x x$ component of the Cauchy stress tensor, $\left\langle\sigma_{x x}\right\rangle$. For frictionless particles $\left(\mu_{\mathrm{f}}=0\right),\left\langle\sigma_{y y}\right\rangle \simeq\left\langle\sigma_{x x}\right\rangle$. This means that like in a liquid at rest, the vertical component of the force applied at the mobile wall is integrally redistributed in any direction. This liquid-like behavior for the Cauchy stress tensor is not valid anymore in the case of the frictional particles, and $\left\langle\sigma_{y y}\right\rangle$ is then larger than $\left\langle\sigma_{x x}\right\rangle$. Indeed, for the frictional particles, the non isotropic (uni-axial) loading of the system leads to an anisotropic deformation of the particles. The latter is averagely more pronounced in the loading direction ( $y$ direction). This can be observed in Fig. 5, in which the mean value of $D_{x} / D_{y}$ for all

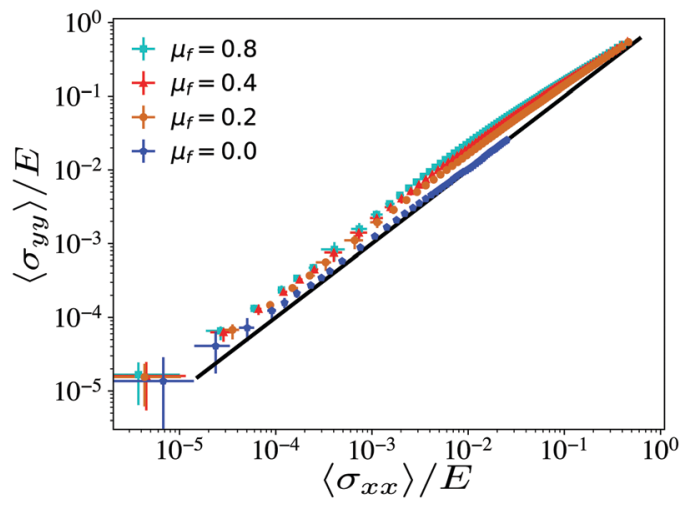

Fig. 4 Mean value of the $y y$ component of the Cauchy stress tensor, $\left\langle\sigma_{y y}\right\rangle$, as a function of the mean value of the $x x$ component of the Cauchy stress tensor, $\left\langle\sigma_{x x}\right\rangle$, for the four coefficients of friction $\mu_{\mathrm{f}}$. The error bars are determined by the statistical error of the three initial configurations.

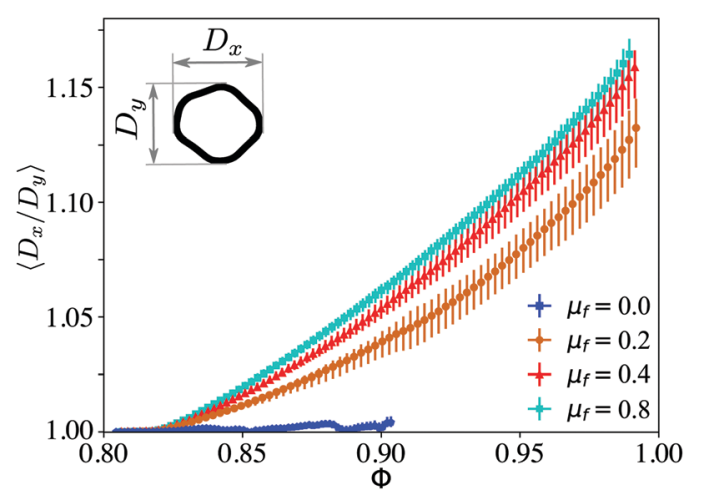

Fig. 5 Mean value of the ratio between the $y$-projection length of a deformed particle $\left(D_{y}\right)$ and its $x$-projection $\left(D_{x}\right)$ as a function of the packing fraction $\Phi$ for the four coefficients of friction $\mu_{\mathrm{f}}$. The error bars are determined by the statistical error of the three initial configurations.

particles (where $D_{y}$ is the $y$-projection length of a deformed particle and $D_{x}$ its $x$-projection) is presented as a function of the packing fraction $\Phi$.

Note that the mean value of the $x y$ component of the Cauchy stress tensor, $\left\langle\sigma_{x y}\right\rangle$, is equal to zero due to the $x \leftrightarrow-x$ symmetry of the system.

\section{B. Spatial distribution of the stress}

Three snapshots of the uni-axial compression of a system with the coefficient of friction of $\mu_{\mathrm{f}}=0.2$ are shown in Fig. 6 for different packing fractions: before the rigidity transition, $\Phi=0.804$ (Fig. 6(a)), after the rigidity transition, $\Phi=0.86$ (Fig. 6(b)), and at high packing fraction, $\Phi=0.98$ (Fig. $6(\mathrm{c})$ ). The gray scales indicate the values of $\sigma_{y y} / E$ inside the particles. The force chains appearing at the rigidity transition are here observable in terms of stresses. ${ }^{45}$ They materialize the strong heterogeneity of the stress distribution inside the system.

The probability density functions (pdf) of $\sigma_{y y} /\left\langle\sigma_{y y}\right\rangle$ is shown in Fig. 7 for several packing fractions and $\mu_{f}=0.2$. As expected, $\sigma_{y y}$ can be positive (the matter being stretched) or negative (the matter being squeezed). We also observe that the maximum of 


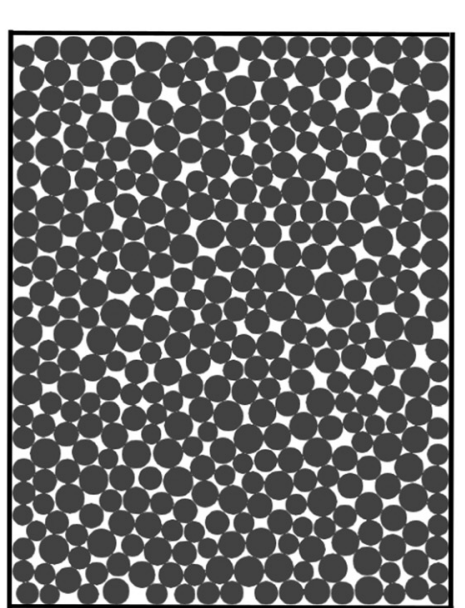

(a)

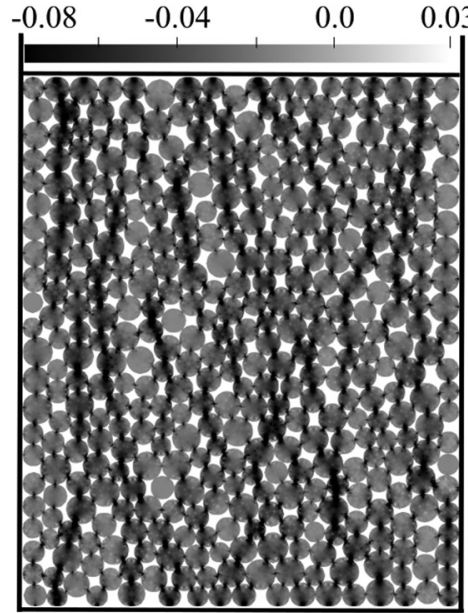

(b)

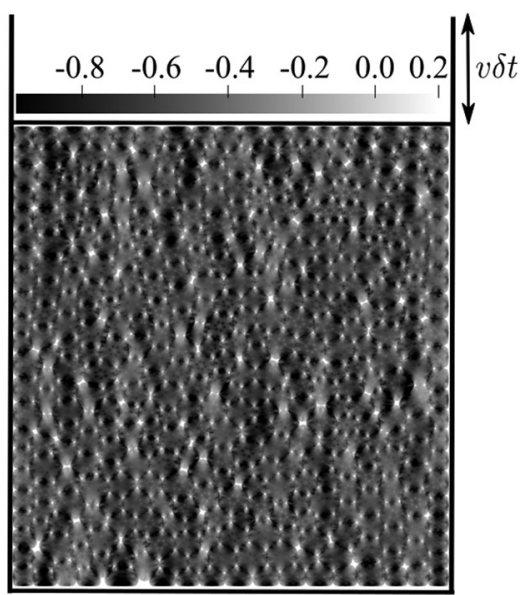

(c)

Fig. 6 Three snapshots of the compaction of the granular system with the coefficient of friction of $\mu_{\mathrm{f}}=0.2$ : (a) $\Phi=0.804$, (b) $\Phi=0.86$ and (c) $\Phi=0.98$. The gray level indicates the values of the $y y$ component of the Cauchy stress tensor, divided by the Young's modulus of the particles, $\sigma_{y y} / E$.

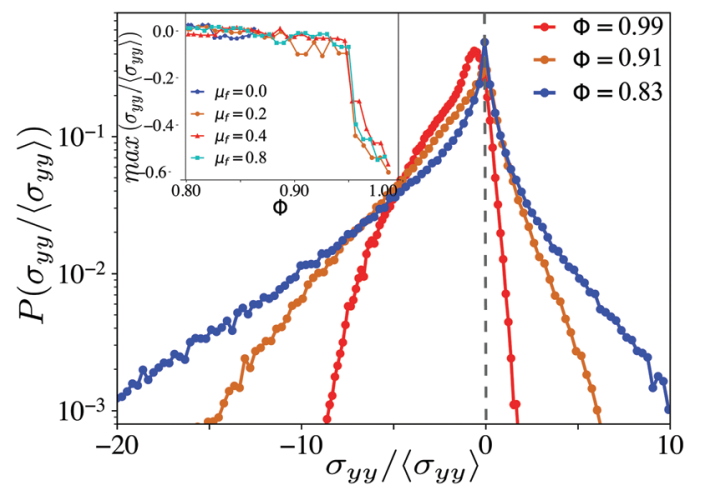

Fig. 7 Probability density function of $\sigma_{y y} /\left\langle\sigma_{y y}\right\rangle$ for three packing fractions and for $\mu_{\mathrm{f}}=0.2$. The data come from three initial configurations. Inset: Most probable value of $\sigma_{y y} /\left\langle\sigma_{y y}\right\rangle$ as a function of the packing fraction $\Phi$ for several values of the coefficient of friction.

the pdf is for $\sigma_{y y} \simeq 0$ at low packing fractions, $\Phi<0.94$; see inset of Fig. 7. For larger packing fractions, the maximum of this pdf is for a non zero negative value of $\sigma_{y y}$, whose absolute value rapidly increases with $\Phi$. Hence, for $\Phi<0.94$ the most common value of $\sigma_{y y}$ within the particles is zero, while for $\Phi>0.94$, the most probable value of $\sigma_{y y}$ is smaller than zero. The same behavior is also observed for the other values of the coefficient of friction.

A global measure of the heterogeneities in the distribution of $\sigma_{y y}$ can be obtained by computing the coefficient of variation (or the relative standard deviation) of $\sigma_{y y}$, defined as: ${ }^{46}$

$$
C_{v}\left(\sigma_{y y}\right)=\frac{\left\langle\sigma_{y y}{ }^{2}\right\rangle-\left\langle\sigma_{y y}\right\rangle^{2}}{\left\langle\sigma_{y y}\right\rangle^{2}} .
$$

Fig. 8 presents $C_{v}\left(\sigma_{y y}\right)$ as a function of $\Phi$. Note that the values of $C_{v}\left(\sigma_{y y}\right)$ are only computed for $\Phi \geq \Phi_{J}$ since $\left\langle\sigma_{y y}\right\rangle$ is nearly equal to zero for the packing fractions lower than $\Phi_{J}$. The error bars denote the statistical variability of $C_{v}\left(\sigma_{y y}\right)$ for the different initial configurations. For any values of the coefficient of friction, $C_{v}\left(\sigma_{y y}\right)$ decreases with $\Phi$. This is compatible with Fig. 6: the distribution of $\sigma_{y y}$ is more dispersed near the rigidity transition (Fig. 6(b)) compared to the higher packing fractions (Fig. 6(c)). This is also observed in the previous work, ${ }^{25}$ for the coefficient of variation of the strain energy density, but in a smaller granular system (100 particles), for a unique value of the coefficient of friction and for a limited range of the packing fractions $(\Phi<0.88)$. Interestingly, $C_{v}\left(\sigma_{y y}\right)$ exhibits an inflection point near the packing fraction delimiting the two regimes evidenced in Section III. Furthermore, for a given value of the packing fraction, $C_{v}\left(\sigma_{y y}\right)$ significantly depends on the coefficient of friction: the distribution of $\sigma_{y y}$ is more spread out (i.e. more heterogeneous) when the coefficient of friction increases; see inset of Fig. 8. This is because the friction prevents the particle rearrangements, leading to large deformations of the particles under compression and hence, to more pronounced heterogeneities inside the particles.

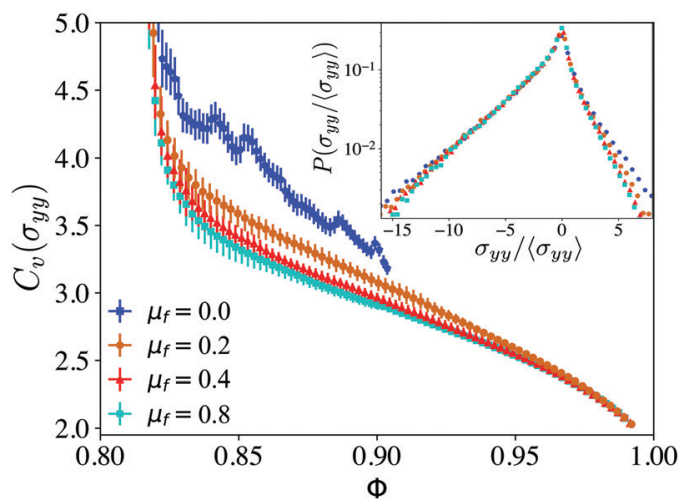

Fig. 8 Coefficient of variation of $\sigma_{y y}$ as a function of the packing fraction $\Phi$ for four different coefficients of friction $\mu_{\mathrm{f}}$. The error bars correspond to the variation of this mean value for the three initial configurations. Inset: Probability density function of $\sigma_{y y} /\left\langle\sigma_{y y}\right\rangle$ for the packing fraction of $\Phi=0.86$ and for four different coefficients of friction $\mu_{\mathrm{f}}$. 


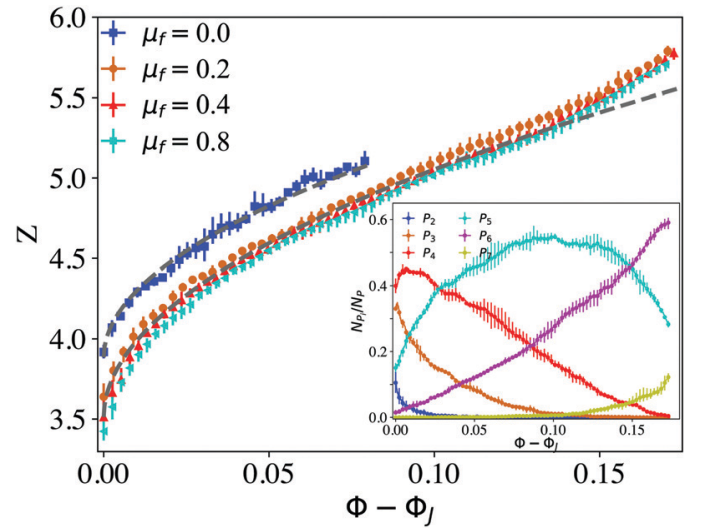

Fig. 9 Mean coordination number $Z$ as a function of the excess packing fraction $\Phi-\Phi_{J}$ for four coefficients of friction. The error bars correspond to the variation of $Z$ for the three initial configurations. The dashed lines are the power-law fit; see eqn (4). Inset: Proportion of particles with 2, 3, 4, 5,6 and 7 contacts with other particles (resp. $N_{P 2}, N_{P 3}, N_{P 4}, N_{P 5}, N_{P 6}, N_{P 7}$ ) in the granular system with the coefficient of friction of $\mu_{\mathrm{f}}=0.2$.

The decrease of $C_{v}\left(\sigma_{y y}\right)$ with the packing fraction can be explained by the evolution of the micro-structure. During the compaction, the particles are more and more deformed and then, the average contact length between the particles increases. The stress at inter-particles contacts is therefore more regular while the packing fraction increases. In addition, due to these deformations, the average coordination number $Z$, defined as the average number of contact neighbors per particle, increases (Fig. 9). The contact forces are then more and more equally distributed along the perimeter of the particles, which explains why $C_{v}\left(\sigma_{y y}\right)$ decreases as the packing fraction increases. Note that the fraction of the particles with 7 neighbors starts to increase from $\Phi \simeq 0.93$ (see inset of Fig. 9) i.e. at the packing fraction delimiting the two regimes previously illustrated. With the size polydispersity of the system, the particles with 7 neighbors require large deformations so that they can be partially embedded in the remaining spaces between their neighbors. This shows that the sneaking of the squeezed parts of the particles inside the empty spaces between the particles plays an important role in the final regime of the compaction $(\Phi>0.93)$. Furthermore, the coordination number is found to increase as a power-law with the packing fraction: ${ }^{12,17,44}$

$$
Z-Z_{J} \propto \sqrt{\Phi-\Phi_{J}},
$$

with $Z_{J}$ corresponding to $\Phi_{J}$, and for low to moderate values of $\Phi-$ $\Phi_{J}$ (see Fig. 9). This relation was observed in many other soft particulate systems like foams or emulsions. ${ }^{12,44}$ For the packing fractions larger than $\Phi \simeq 0.93$, the coordination number increases faster than $\sqrt{\Phi-\Phi_{J}}$. This result may be due to the very large deformation of the particles, making possible the occurrence of new contacts.

\section{Plasticity}

\section{A. Irreversibility at the global scale}

The evolution of the mean stress $\left\langle\sigma_{y y}\right\rangle$ as a function of $\Phi$, during the compressive loading and unloading by the constant

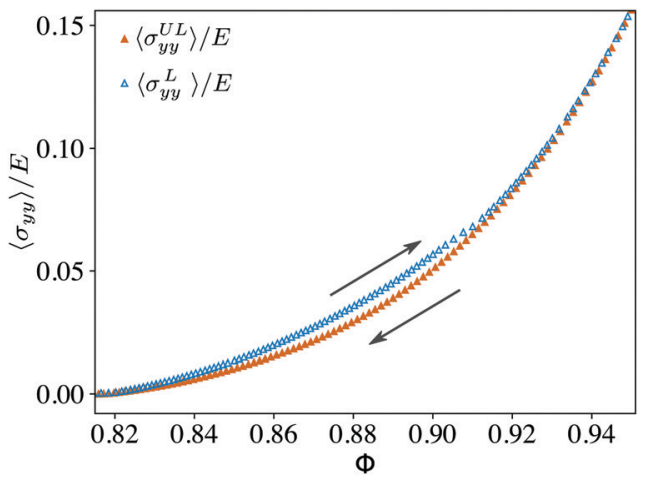

Fig. 10 Evolution of the mean stress normalized by the Young's modulus, $\left\langle\sigma_{y y}\right\rangle / E$, as a function of the packing fraction $\Phi$ during the compressive loading up to $\Phi=0.95$ and then, unloading from this packing fraction. The applied velocity for loading and unloading is equal to $0.035 \mathrm{~m} \mathrm{~s}^{-1}$ and the coefficient of friction is $\mu_{\mathrm{f}}=0.2$.

velocity of $v=0.035 \mathrm{~m} \mathrm{~s}^{-1}$, is shown in Fig. 10 for a granular system with the coefficient of friction of $\mu_{\mathrm{f}}=0.2$. Indeed, the system is first subjected to a compressive loading up to the packing fraction of $\Phi=0.95$, before being gradually and completely unloaded. $\left\langle\sigma_{y y}\right\rangle$ is smaller during the decompaction than during the compaction for a given value of $\Phi$ (smaller than 0.95). As a consequence, the work done by the force applied to the mobile wall during loading is larger than the energy released during unloading. For a quasi-static compaction (i.e. in the limit of infinitely small velocities of the mobile wall as it is the case here), the dissipation due to the (Rayleigh) damping is expected to be negligible. The remaining source of energy dissipation is hence the inter-particles friction, except in two cases for which there is no frictional energy dissipation: particles without friction $\left(\mu_{\mathrm{f}}=0\right)$ or with very large coefficients of friction. In the last case, this occurs because there is no interparticles sliding and, hence no particle rearrangement.

The relative difference between loading stress, $\left\langle\sigma_{y y}^{\mathrm{L}}\right\rangle$, and unloading stress, $\left\langle\sigma_{y y}^{\mathrm{UL}}\right\rangle$, for four coefficients of friction and for several values of the packing fraction corresponding to the unloading points ( $\Phi=0.85,0.90$ and 0.95), is shown in Fig. 11(a). We observe that these relative differences are not null, and even, for $\mu_{\mathrm{f}}=0$ (no frictional dissipation), the relative difference of the stress is the highest and declines when the coefficient of friction increases; see Fig. 11(a). We hence observe that the energy dissipation is more important when the inter-particles friction is smaller in the granular systems.

In order to go further let us consider now, for the compressive loading, the evolution of non-affine displacement field $u_{\text {n.a }}(\mathbf{r})$, i.e. the non-homogeneous part of the displacement field of the material points of the system. ${ }^{47} u_{\text {n.a }}(\mathbf{r})$ is computed by considering an interval displacement $v \Delta t$ of the mobile wall (with $\Delta t$ as the elapsed time of the interval). Here, the interval displacement is set to be $v \Delta t=1.14 \times 10^{-4} \mathrm{~m}$. The mean non-affine displacement $\left\langle u_{\text {n.a }}\right\rangle$ normalized by $v \Delta t$, is displayed as a function of the packing fraction $\Phi$ in Fig. 11(b) for four coefficients of friction. $\left\langle u_{\text {n.a }}\right\rangle /(v \Delta t)$ fluctuates close to zero with some major peaks. The mean nonaffine displacement is found to be higher when the coefficient of 


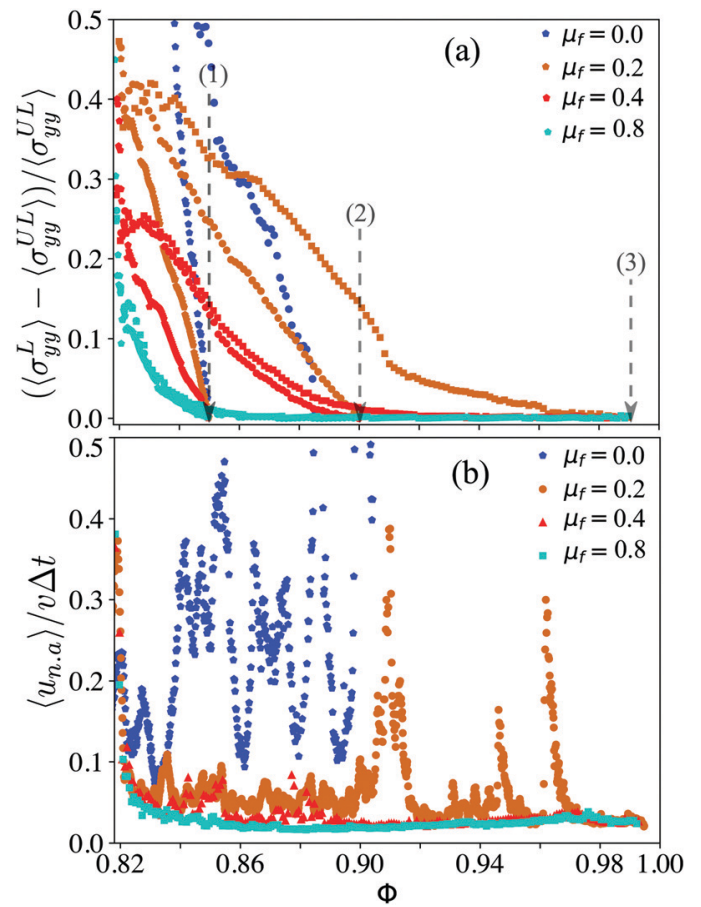

Fig. 11 (a) Relative difference of $\left\langle\sigma_{y y}\right\rangle$ between the compressive loading and unloading as a function of the packing fraction $\Phi$ for four coefficients of friction and for several packing fractions corresponding to the unloading points ( $\Phi=0.85$ (1), 0.9 (2) and 0.95 (3)); (b) mean non-affine displacement $\left\langle u_{\text {n.a }}\right\rangle$ normalized by an interval displacement $v \Delta t\left(v \Delta t=1.14 \times 10^{-4} \mathrm{~m}\right)$ as a function of the packing fraction $\Phi$ for four coefficients of friction.

friction is smaller. It is interesting to note that these peaks are correlated with the sudden changes in the values of $\left\langle\sigma_{y y}^{\mathrm{L}}\right\rangle-\left\langle\sigma_{y y}^{\mathrm{UL}}\right\rangle$, presented in Fig. 11(a). We thus observe a correlation between the mean non-affine displacement and the energy dissipation during the compressive loading and unloading of the granular system, seen above.

In order to get insight on the nature of these non affine displacements, we examine the non-affine displacement distribution over the whole system. The high values in the non-affine displacement map is found to be spatially localized; see Fig. 12. It consists in a local reorganization (referred later as an elementary event), occurring in a short time step and involving few particles. The occurred elementary event is centered on an inter-particles contact point. We observe (if the event is not located too close to the cell boundaries) quasi-fourfold distributions of the non-affine displacement field, similar to the stress relaxation associated to Eshelby's inclusions; ${ }^{48}$ see Fig. 12. During an elementary event, the system suddenly releases a part of the strain energy. In the energy landscape of the whole system, the system leaves a local minimum energy state and finds a lower energy state. The corresponding finite difference of the energy is first converted into the kinetic energy that will be dissipated through damping. Similar mechanisms of sudden change on the local minimum in the energy landscape of a system was demonstrated in attractive systems as metallic glasses, ${ }^{4-51}$ soft glassy materials, ${ }^{47,52,53}$ and compressed granular materials in simple shear geometry and for non deformable particles. ${ }^{54}$

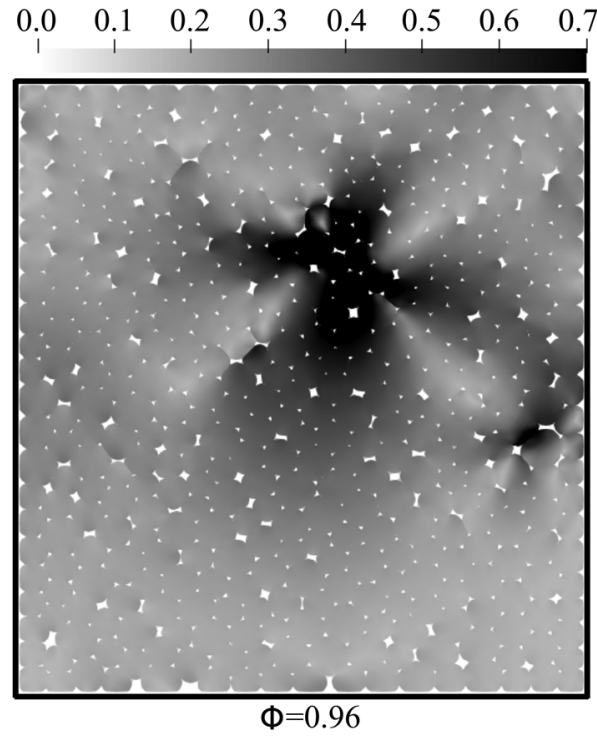

Fig. 12 Snapshot of non-affine displacement distribution in the granular system with the coefficient of friction of $\mu_{\mathrm{f}}=0.2$ for the packing fraction of $\Phi=0.96$. The gray scale indicates the magnitude of the non affine displacement normalized by $v \Delta t\left(v \Delta t=1.14 \times 10^{-4} \mathrm{~m}\right)$.

To summarize, the main source of the energy dissipation in the granular system is not friction, and the energy can be dissipated through elementary events. The energy dissipation is more important when the friction coefficient is lower. Since the sliding of particles generates elementary events, the interparticles friction minimizes these events by reducing the sliding. A large coefficient of friction hence reduces the plastic features of the compressed granular system.

\section{Concluding remarks}

The uni-axial compression of two-dimensional athermal granular assemblies composed of incompressible neo-Hookean elastic particles has been investigated numerically, with a special focus on the inter-particles friction effects. Once the applied stress is of the order of the elastic modulus of the particles, the deformations of the particles exceed the well-studied limit of the semi-rigid particles, and packing fractions close to 1 can be achieved. In this regime, the initially circular particles become faceted, and the mean coordination number tends to the value of 6 and even, some particles have more than 6 neighbors. The stress distribution in the granular system becomes more and more homogeneous as the packing fraction increases beyond the rigidity transition. It means that the discrete features of the granular system are less and less pronounced.

Irreversible features during the compaction were demonstrated. They result from elementary events consisting in the localized sliding of particles, and are reminiscent to what is observed in other plastic systems as soft glassy materials or metallic glasses. The friction effect on the energy dissipation in the compressed granular assemblies at large packing fraction was highlighted. For large packing fractions, irreversibility is mainly driven by elasticity because the strain energy stored in the particles is far 
larger than the energy dissipated by friction. The stabilizing effect of friction reduces the occurrences of elementary events and thus, lowers energy dissipation. We did not address here the case of elementary events occurring in systems close to the rigidity transition. In such configurations, the friction effect is expected to be higher due to particle movements; and because of the small deformation of particles, the strain energy stored in them is lower. This limit will be investigated in a future work.

The long-ranged interactions between elementary events are known to redistribute the stress in the material, which is at the origin of formation of spatial heterogeneities (e.g. shear bands) at the macroscopic scale. ${ }^{53,55,56}$ Our system is too small to study such interactions. The recent observation of quadrupolar Eshelby-like stress distribution in granular systems ${ }^{54}$ is an indication that the plastic behavior of soft granular systems could be described in the framework of plasticity theory of soft glasses plasticity. The study of neo-Hookean particle assemblies with larger number of particles but with a looser mesh density, will be the subject of a forthcoming study with the aim of analyzing the interactions between different plastic events and the resulting spatial heterogeneities for different coefficients of friction.

\section{Conflicts of interest}

There are no conflicts to declare.

\section{Acknowledgements}

The authors thank Farhang Radjai for highlighting discussions.

\section{References}

1 W. R. Ketterhagen, M. T. am Ende and B. C. Hancock, Process Modeling in the Pharmaceutical Industry using the Discrete Element Method, J. Pharm. Sci., 2009, 98, 442-470.

2 W. R. Ketterhagen, Modeling the motion and orientation of various pharmaceutical tablet shapes in a film coating pan using DEM, Int. J. Pharm., 2011, 409, 137-149.

3 A. J. Rogers, A. Hashemi and M. G. Ierapetritou, Modeling of Particulate Processes for the Continuous Manufacture of Solid-Based Pharmaceutical Dosage Forms, Processes, 2013, 1, 67-127.

4 C. L. Martin, Elasticity, fracture and yielding of cold compacted metal powders, J. Mech. Phys. Solids, 2004, 52.

$5 \mathrm{H}$. A. Häggblad and M. Oldenburg, Modelling and simulation of metal powder die pressing with use of explicit time integration, Modell. Simul. Mater. Sci. Eng., 1994, 2, 893-911.

6 H. Chtourou, M. Guillot and A. Gakwaya, Modeling of the metal powder compaction process using the cap model. Part I. Experimental material characterization and validation, Int. J. Solids Struct., 2002, 39, 1059-1075.

7 P. Pizette, C. Martin, G. Delette, P. Sornay and F. Sans, Compaction of aggregated ceramic powders: From contact laws to fracture and yield surfaces, Powder Technol., 2010, 198, 240-250.
8 Y. Gan and M. Kamlah, Discrete element modelling of pebble beds: With application to uniaxial compression tests of ceramic breeder pebble beds, J. Mech. Phys. Solids, 2010, 58, 129-144.

9 A. R. Boccaccini and Z. Fan, A new approach for the Young's modulus-porosity correlation of ceramic materials, Ceram. Int., 1997, 23, 239-245.

10 C. L. Martin, D. Bouvard and G. Delette, Discrete Element Simulations of the Compaction of Aggregated Ceramic Powders, J. Am. Ceram. Soc., 2006, 89, 3379-3387.

11 C. S. O'Hern, L. E. Silbert, A. J. Liu and S. R. Nagel, Jamming at zero temperature and zero applied stress: The epitome of disorder, Phys. Rev. E: Stat., Nonlinear, Soft Matter Phys., 2003, 68, 011306.

12 M. van Hecke, Jamming of soft particles: geometry, mechanics, scaling and isostaticity, J. Phys.: Condens. Matter, 2010, 22, 033101.

13 R. T. Bonnecaze and M. Cloitre, Micromechanics of soft particle glasses, Adv. Polym. Sci., 2010, 236, 117-161.

14 A. Favier de Coulomb, M. Bouzid, P. Claudin, E. Clément and B. Andreotti, Rheology of granular flows across the transition from soft to rigid particles, Phys. Rev. Fluids, 2017, 2, 102301.

15 S. Nezamabadi, T. H. Nguyen, J. Y. Delenne and F. Radjai, Modeling soft granular materials, Granular Matter, 2017, $19,8$.

16 S. Nezamabadi, F. Radjai, J. Averseng and J. Y. Delenne, Implicit frictional-contact model for soft particle systems, J. Mech. Phys. Solids, 2015, 83, 72-87.

17 S. Nezamabadi, X. Frank, J. Y. Delenne, J. Averseng and F. Radjai, Parallel implicit contact algorithm for soft particle systems, Comput. Phys. Commun., 2019, 237, 17-25.

18 J. J. Moreau, Some numerical methods in multibody dynamics: application to granular materials, European Journal of Mechanics A Solids, 1994, 13, 93-114.

19 F. Radjai and F. Dubois, Discrete-element Modeling of Granular Materials, London, Iste-Wiley, 2011.

20 A. A. Munjiza, D. R. J. Owen and N. Bicanic, A combined finite-discrete element method in transient dynamics of fracturing solids, Engineering Computations, 1995, 12, 145-174.

21 A. A. Munjiza, The Combined Finite-Discrete Element Method, Wiley, Chichester, 2004.

22 D. T. Gethin, R. S. Ransing, R. W. Lewis, M. Dutko and A. J. L. Crook, Numerical comparison of a deformable discrete element model and an equivalent continuum analysis for the compaction of ductile porous material, Comput. Struct., 2001, 79, 1287-1294.

23 R. W. Ogden, Non-Linear Elastic Deformations, Ellis Horwood Limited, Chichester, 1984.

24 T. L. Vu, S. Nezamabadi, J. Barés and S. Mora, Analysis of dense packing of highly deformed grains, EPJ Web of Conferences., 2017, 140, 15031.

25 T. L. Vu, J. Barés, S. Mora and S. Nezamabadi, Numerical simulations of the compaction of assemblies of rubberlike particles: A quantitative comparison with experiments, Phys. Rev. E, 2019, 99, 062903. 
26 C. P. Goodrich, A. J. Liu and S. R. Nagel, Finite-Size Scaling at the Jamming Transition, Phys. Rev. Lett., 2012, 109, 095704.

27 S. Mora, C. Maurini, T. Phou, J. M. Fromental, B. Audoly and Y. Pomeau, Solid drops: Large capillary deformations of immersed elastic rods, Phys. Rev. Lett., 2014, 113, 178301.

28 S. Mora and Y. Pomeau, Softening of edges of solids by surface tension, J. Phys.: Condens. Matter, 2015, 27, 194112.

29 K. L. Johnson and K. Kendall, Surface Energy and the Contact of Elastic Solids, Proc. R. Soc. London, Ser. A, 1971, 324, 301.

30 LMGC90. LMGC90; 2018. https://git-xen.lmgc.univ-montp2. fr/lmgc90/lmgc90_user/wikis/home.

31 C. Lozano, G. Lumay, I. Zuriguel, R. Hidalgo and A. Garcimartin, Breaking Arches with Vibrations: The Role of Defects, Phys. Rev. Lett., 2012, 109, 068001.

32 R. Artoni, A. Soligo, J. Paul and P. RIchard, Shear localization and wall friction in confined dense granular flows, J. Fluid Mech., 2018, 849, 395-418.

33 S. Nezamabadi, H. Zahrouni and J. Yvonnet, Solving hyperelastic material problems by asymptotic numerical method, Comput. Mech., 2011, 47, 77-92.

34 T. Vu, J. Barés, S. Mora and S. Nezamabadi, Deformation field in diametrically loaded soft cylinders, Exp. Mec., 2019, 59, 453-467.

35 P. Olsson and S. Teitel, Critical Scaling of Shear Viscosity at the Jamming Transition, Phys. Rev. Lett., 2007, 99, 178001.

36 V. J. Langlois, S. Hutzler and D. Weaire, Rheological properties of the soft-disk model of two-dimensional foams, Phys. Rev. E: Stat., Nonlinear, Soft Matter Phys., 2008, 78, 021401.

37 G. Katgert and M. van Hecke, Jamming and geometry of two-dimensional foams, Europhys. Lett., 2010, 92, 34002.

38 D. A. Head, Critical Scaling and Aging in Cooling Systems Near the Jamming Transition, Phys. Rev. Lett., 2009, 102, 138001.

39 T. Majmudar, M. Sperl, S. Luding and R. Behringer, Jamming transition in granular systems, Phys. Rev. Lett., 2007, 98, 058001.

40 O. Dauchot, G. Marty and G. Biroli, Dynamical Heterogeneity Close to the Jamming Transition in a Sheared Granular Material, Phys. Rev. Lett., 2005, 95, 265701.

41 A. S. Keys, A. R. Abate, S. C. Glotzer and D. J. Durian, Measurement of growing dynamical length scales and prediction of the jamming transition in a granular material, Nature, 2007, 3, 260.

42 L. Berthier and G. Biroli, Theoretical perspective on the glass transition and amorphous materials, Rev. Mod. Phys., 2011, 83, 587-645.
43 T. Majmudar and R. Behringer, Contact force measurements and stress-induced anisotropy in granular materials, Nature, 2005, 435, 7045.

44 C. S. O'Hern, A. L. Langer, A. J. Liu and S. R. Nagel, Random Packings of Frictionless Particles, Phys. Rev. Lett., 2002, 88, 075507.

45 F. Radjai, M. Jean, J. Moreau and S. Roux, Force distributions in dense two-dimensional granular systems, Phys. Rev. Lett., 1996, $77,274$.

46 W. Hofmann, B. Asgharian, R. Bergmann, S. Anjilvel and F. W. Miller, The effect of heterogeneity of lung structure on particle deposition in the rat lung, Toxicol. Sci., 2000, 532, 430-437.

47 C. Maloney and A. Lemaitre, Amorphous systems in athermal, quasistatic shear, Phys. Rev. E: Stat., Nonlinear, Soft Matter Phys., 2006, 74, 016118.

48 J. D. Eshelby, The determination of the elastic field of an ellipsoidal inclusion, and related problems, Proc. R. Soc. London, Ser. A, 1957, 241, 376.

49 A. Argon, Plastic deformation in metallic glasses, Acta Metall., 1979, 27, 47-58.

50 A. Argon and H. Kuo, Plastic flow in a disordered bubble raft (an analog of a metallic glass), Mater. Sci. Eng., 1979, 39, 101-109.

51 M. L. Falk and J. S. Langer, Dynamics of viscoplastic deformation in amorphous solids, Phys. Rev. E: Stat. Phys., Plasmas, Fluids, Relat. Interdiscip. Top., 1998, 57, 7192.

52 P. Schall, D. Weitz and F. Spaepen, Structural Rearrangements That Govern Flow in Colloidal Glasses, Science, 2007, 318, 1895-1899.

53 F. Puosi, J. Rottler and J. L. Barrat, Time-dependent elastic response to a local shear transformation in amorphous solids, Phys. Rev. E: Stat., Nonlinear, Soft Matter Phys., 2014, 89, 042302.

54 S. McNamara, J. Crassous and A. Amon, Eshelby inclusions in granular matter: Theory and simulations, Phys. Rev. E, 2016, 94, 022907.

55 A. Tanguy, F. Leonforte and J. L. Barrat, Plastic response of a 2D Lennard-Jones amorphous solid: Detailed analysis of the local rearrangements at very slow strain rate, Eur. Phys. J. E: Soft Matter Biol. Phys., 2006, 20, 355-364.

56 F. Puosi, J. Rottler and J. L. Barrat, Plastic response and correlations in athermally sheared amorphous solids, Phys. Rev. E, 2016, 94, 032604. 